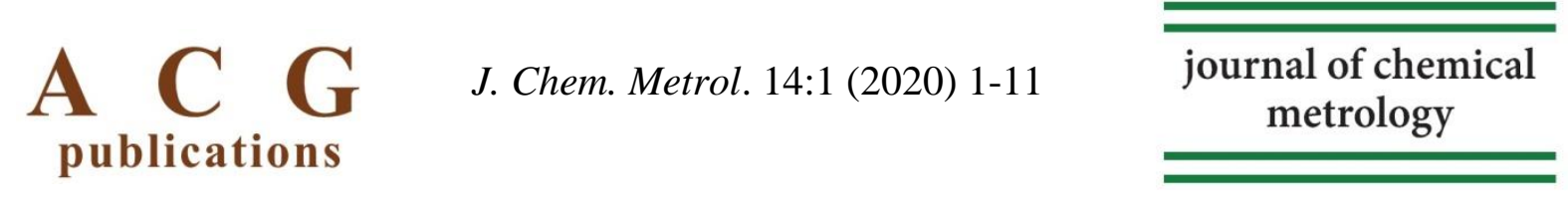

\title{
Rapid and easy method for simultaneous measurement of widespread 27 compounds in natural products and foods
}

\author{
Özge Tokul-Ölmez $\odot$, Bihter Şahin $\odot$, Cansel Çakır $\odot$ and \\ Mehmet Öztürk
}

\author{
Muğla Sitku Koçman University, Faculty of Science, Department of Chemistry, 48121 Menteşe, \\ Muğla, Türkiye
}

(Received March 27, 2020; Revised April 23, 2020; Accepted April 26, 2020)

\begin{abstract}
Phenolic compounds are dietary bioactive compounds that are available in foods and beverages. They are produced by the plant and/or by the mushrooms, naturally. Some of the natural phenolic compounds were also used in dietary supplements in the pharmaceutical or food industries. For simultaneous analyses of phenolic compounds in one injection, a rapid and sensitive method was developed for twenty-seven natural compounds using reversephase high-performance liquid chromatography (RP-HPLC) coupled with diode array detector (DAD). The linear gradient elution systems of $0.1 \%$ acetic acid-methanol were used, and the analyzing time was 61 minutes. The method was validated with linearity, relative error, reproducibility, LOD values. Relative standard deviation between $(n=7)$ within and between days for reproducibility is less than $10 \%$, and relative error (or recovery) is less than 5\%. The method exhibited excellent linearity $\left(R^{2}>0.999\right)$, good precision $(\mathrm{RSD}<6.1 \%)$, recovery $(97.6-104.1 \%)$ and limits of detection $(0.23-28.81 \mu \mathrm{g} / \mathrm{m} 1)$ and quantification $(1.62-87.29 \mu \mathrm{g} / \mathrm{m} 1)$. The detection of compounds was performed at $220 \mathrm{~nm}, 280 \mathrm{~nm}$, and $330 \mathrm{~nm}$. The developed method for the rapid determination of phenolic compounds using RP-HPLC can be used to identify the availability of these phenolic compounds in natural and commercial products.
\end{abstract}

Keywords: RP-HPLC; phenolic compounds, food; dietary supplements; natural products. (C) 2020 ACG Publications. All rights reserved.

\section{Introduction}

The plants produce phenolic compounds to protect themselves against ultraviolet radiation or pathogenic aggression. Phenolic compounds are considered as secondary metabolites except for some of them. Plants contain bioactive phenolic compounds. Previous studies revealed that phenolic compounds possess antioxidant, anticancer, antimicrobial, anticholinesterase, anti-urease, and anti-tyrosinase activities. They are also known as antipyretic, biogenic, analgesic, antibiotic, and sedative properties [13]. Therefore, the plants containing bioactive phenolic compounds are used to prepare food supplements. Moreover, mainly they exhibit antioxidant activity. Therefore, to screen them in foods and medicinal plants, many studies have been conducted.

Many different methods have been developed for determining the phenolic and organic components in natural products [4]. For this purpose, the best instrument is HPLC (high-performance liquid chromatography), which can be successfully applied for analyzing chemical compounds of natural products [5]. Thirteen phenolic compounds were also used to develop the HPLC method [6]. In another

\footnotetext{
${ }^{*}$ Corresponding author E-Mail: mehmetozturk@ mu.edu.tr
} 
developed method, nineteen phenolic and flavonoid derivatives were simultaneously analyzed using HPLC-DAD and fluorescence detector using a fused-core type column in 10 minutes [7]. The phenolics of Manzanilla olives collected from various regions in Spain were analyzed using this method, where ODS was the column for separation in the extracts obtained by the different extraction techniques [8]. There is another study to analyze twenty-five phenolic compounds using HPLC-DAD was developed [9]. Herein, beside the retention times, three different wavelengths $(254,280$ and $330 \mathrm{~nm})$ were used to accurate analyses. The method was used to identify the phenolic compounds of grapes. Such an analysis was also developed by [10] using different columns, which is faster and having high resolution. In this HPLC assay beside retention times 220, 280, 320, and $520 \mathrm{~nm}$ wavelengths were used to determine accurately. Thirty phenolic compounds using a phenyl-hexyl-fixed phase, and two detectors were separated in a developed method [11]. The separation took place in 64 minutes, and the resolution and the accuracy were accepted. In another study, 13 phenolic compounds were investigated in the water extracts of Dianthus carmelitarum using reverse-phase column and acetonitrile-water gradient elution system. The analyses took 36 minutes [12].

The literature survey indicated that to analyze the phenolic compounds, abundantly, Diode Array Detector (DAD) was employed. Beside, Florescence, PDA, ECD, and Refractive Index detectors were also used. For the separation of phenolic compounds, in general, the ODS $\left(\mathrm{C}_{18}\right)$ column in $(4.6 \mathrm{~mm} \times 250$ $\mathrm{mm}$, ID, $5 \mu \mathrm{m}$ ) was used. The mobile phase mainly consists of $0.5-3 \%$ organic or inorganic acid, such as acetic acid or phosphoric acid in water and/or methanol/acetonitrile [11, 13-22].

Almost in many laboratories, there is an HPLC coupled with DAD or UV detectors and a standard ODS column. Therefore, by changing some parameters, 27 phenolic compounds mostly available in plant extracts were used to develop a fast and reliable method, simultaneously using HPLC as an instrument. This study aimed to develop an HPLC method that could be easily adapted related studies. The fumaric acid, gallic acid, trans-aconitic acid, p-benzoquinone, pyrocatechol, protocatechuic acid, 2-(4hydroxyphenyl) ethanol, 4-hydroxybenzoic acid, catechin, methyl-p-benzoquinone, rosmarinic acid, quercetin, naringenin, trans-cinnamic acid, ellagic acid, rutin, trans-2-hydroxycinnamic acid, coumarin, ferulic acid, p-coumaric acid, chlorogenic acid, 2,4-dihydroxybenzoic acid, vanillin, caffeic acid, 6,7dihydroxy coumarin, vanillic acid, chrysin which are available in plant samples were used for separation in one injection. This method was validated concerning linearity, reproducibility, and accuracy.

\section{Experimental}

\subsection{Chemicals}

Methanol (HPLC, analytical grade) and glacial acetic acid were supplied from Merck (Sternheim, Germany). Fumaric acid ( $\geq 99.0 \%)$, gallic acid ( $\geq 99.0 \%)$, trans-aconitic acid ( $\geq 98.0 \%), p$-benzoquinone ( $\geq 98.0 \%$ ), pyrocatechol ( $\geq 99.5 \%$ ), protocatechuic acid ( $\geq 99.9 \%)$, 2-(4-hydroxyphenyl) ethanol ( $\geq 98.0 \%)$, 4-hydroxybenzoic acid ( $\geq 99.0 \%)$, (+)-catechin hydrate ( $\geq 98.0 \%)$, methyl-p-benzoquinone $(\geq 98.0 \%)$, rosmarinic acid $(\geq 98.0 \%)$, quercetin $(\geq 95.0 \%)$, naringenin $(\geq 98.0 \%)$, trans-cinnamic acid $(\geq 99.0 \%)$, ellagic acid $(\geq 99.0 \%)$, rutin hydrate $(\geq 94.0 \%)$, trans-2-hydroxycinnamic acid ( $\geq 97.0 \%)$, coumarin $(\geq 99.0 \%)$, ferulic acid $(\geq 99.0 \%)$, p-coumaric acid $(\geq 98.0 \%)$, chlorogenic acid $(\geq 99.0 \%), 2,4-$ dihydroxybenzoic acid ( $\geq 97.0 \%)$, vanillin $(\geq 99.0 \%)$, caffeic acid ( $\geq 98.0 \%)$, 6,7-dihydroxy coumarin ( $\geq 98.0 \%)$, vanillic acid $(\geq 97.0 \%)$, chrysin $(\geq 97.0 \%)$ were purchased from Sigma-Aldrich GmbH (Sternheim, Germany). The structures of reference compounds were given in supporting information.

\subsection{Preparation of Stock Solution of Standard Compounds and Mixture}

To detect the exact retention times, $1.0 \mathrm{mg}$ of every compound dissolved in $2 \mathrm{~mL}$ methanol, were injected into the HPLC, individually. To prepare the mix of reference compounds, $10.0 \mathrm{mg}$ fumaric acid, $2.0 \mathrm{mg}$ gallic acid, $8.0 \mathrm{mg}$ trans-aconitic acid, $2.0 \mathrm{mg}$-benzoquinone, $10.0 \mathrm{mg}$ pyrocatechol, $2.0 \mathrm{mg}$ protocatechuic acid, $13.66 \mathrm{mg}$ 2-phenyl ethanol, $0.50 \mathrm{mg}$ 4-hydroxybenzoic acid, $5.0 \mathrm{mg}(+)$-catechin hydrate, $1.0 \mathrm{mg}$ methyl-p-benzoquinone, $2.0 \mathrm{mg}$ 6,7-dihydroxy coumarin, $1.0 \mathrm{mg}$ vanillic acid, $1.2 \mathrm{mg}$ caffeic acid, $1.0 \mathrm{mg}$ vanillin, $1.0 \mathrm{mg}$ 2,4-dihydroxybenzoic acid, $1.0 \mathrm{mg}$ chlorogenic acid, $4.0 \mathrm{mg} p$ - 
coumaric acid, $3.0 \mathrm{mg}$ ferulic acid, $1.0 \mathrm{mg}$ coumarin, $4.0 \mathrm{mg}$ trans-2-hydroxycinnamic acid, $2.0 \mathrm{rutin}$ hydrate, $2.0 \mathrm{mg}$ ellagic acid, $0.50 \mathrm{mg}$ trans-cinnamic acid, $6.2 \mathrm{mg}$ naringenin, $1.0 \mathrm{mg}$ quercetin, $1.2 \mathrm{mg}$ rosmarinic acid, $1.0 \mathrm{mg}$ chrysin were weighted using a balance (Sartorius CPA225D, Gottingen, Germany). Then all compounds weighted accurately were mixed and dissolved in a $10.0 \mathrm{~mL}$ flask with methanol. The mixture was stored at $4{ }^{\circ} \mathrm{C}$ for a maximum period of 2 weeks. From the mixture of 27 compounds stock solutions, eight dilutions were prepared, and each was injected into the HPLC-DAD to prepare the calibration curves of each compound.

\subsection{HPLC Analysis Conditions}

The analyses of 27 compounds were carried out using a Shimadzu high-performance liquid chromatography (Shimadzu Cooperation, Japan) system that consists of a Shimadzu model LC-20AT solvent delivery unit and a Shimadzu model SPD-M20A diode array detection system. All were controlled by LC-solution software (CBM-20A System Controller Shimadzu). The column temperature was set at $35^{\circ} \mathrm{C}$. The chromatographic separation was performed on an Inertsil ODS-3 (4 $\left.\mu \mathrm{m}, 4.0 \mathrm{~mm} \times 150 \mathrm{~mm}\right)$ column and Inertsil ODS-3 guard column. The mobile phases were $0.1 \%$ acetic acid in water (A) and $0.1 \%$ acetic acid in methanol (B). The elution profile was as follows: $2 \% \mathrm{~B}$ in $3 \mathrm{~min}, 2-5 \% \mathrm{~B}$ in $3 \mathrm{~min}$, $5-6 \% \mathrm{~B}$ in $2 \mathrm{~min}, 6-10 \% \mathrm{~B}$ in $4 \mathrm{~min}, 10 \% \mathrm{~B}$ in $1 \mathrm{~min}, 10-25 \% \mathrm{~B}$ in $5 \mathrm{~min}, 25-30 \%$ B in $7 \mathrm{~min}, 30-40 \%$ $\mathrm{B}$ in $5 \mathrm{~min}, 40-42 \% \mathrm{~B}$ in $6 \mathrm{~min}, 42-54 \%$ B in $5 \mathrm{~min}, 54-55 \%$ B in $1 \mathrm{~min}, 55-56 \%$ B in $10 \mathrm{~min}, 56-65 \%$ $\mathrm{B}$ in $4 \mathrm{~min}, 65-75 \% \mathrm{~B}$ in $3 \mathrm{~min}, 75-85 \% \mathrm{~B}$ in $2 \mathrm{~min}, 85-95 \% \mathrm{~B}$ in $5 \mathrm{~min}, 95 \% \mathrm{~B}$ in $2 \mathrm{~min}, 95-100 \% \mathrm{~B}$ in $1 \mathrm{~min}, 100 \% \mathrm{~B}$ in $5 \mathrm{~min}, 100-80 \%$ B in $2 \mathrm{~min}, 80-50 \% \mathrm{~B}$ in $2 \mathrm{~min}, 50-2 \%$ B in $3 \mathrm{~min}$ (Table 1). The flow rate was $1.0 \mathrm{~mL} / \mathrm{min}$. The injected volume was $20 \mu$. Detection was carried out a diode array detector (DAD) using 200-600 nm wavelengths. All the samples and standards were filtered using an Agilent 0.45 $\mu \mathrm{m}$ PTFE filter.

Table 1. The HPLC pump program

\begin{tabular}{rcrc}
\hline Time (Minute) & Solvent B & Minute & Solvent B \\
\hline $0-3(3 \mathrm{~min})$ & $2 \%$ & $43-52(10 \mathrm{~min})$ & $56 \%$ \\
$3-6(3 \mathrm{~min})$ & $5 \%$ & $53-56(4 \mathrm{~min})$ & $65 \%$ \\
$7-8(2 \mathrm{~min})$ & $6 \%$ & $57-59(3 \mathrm{~min})$ & $75 \%$ \\
$9-12(4 \mathrm{~min})$ & $10 \%$ & $60-61(2 \mathrm{~min})$ & $85 \%$ \\
$13(1 \mathrm{~min})$ & $10 \%$ & $62-66(5 \mathrm{~min})$ & $95 \%$ \\
$14-18(5 \mathrm{~min})$ & $25 \%$ & $67-68(2 \mathrm{~min})$ & $95 \%$ \\
$19-25(7 \mathrm{~min})$ & $30 \%$ & $69(1 \mathrm{~min})$ & $100 \%$ \\
$26-30(5 \mathrm{~min})$ & $40 \%$ & $70-74(5 \mathrm{~min})$ & $100 \%$ \\
$31-36(6 \mathrm{~min})$ & $42 \%$ & $75-76(2 \mathrm{~min})$ & $80 \%$ \\
$37-41(5 \mathrm{~min})$ & $54 \%$ & $77-78(2 \mathrm{~min})$ & $50 \%$ \\
$42(1 \mathrm{~min})$ & $55 \%$ & $79-81(3 \mathrm{~min})$ & $2 \%$ \\
\hline
\end{tabular}

\subsection{Method Validation}

The linearity, accuracy, and precision, along with the limit of detection (LOD), the limit of quantitation (LOQ), and effectiveness were considered to validate the reproducibility of the developed method. Various proportions of Solvent A and Solvent B was used to achieve the best resolution. 0.1\% acetic acid in acetonitrile and pure acetonitrile solvents were also used for separation. However, the resolution was unsuccessful for all compounds. The best separation was achieved by using $0.1 \%$ acetic acid in water as a solvent $\mathrm{A}$ and $100 \%$ methanol as a solvent $\mathrm{B}$. On the other hand, the general column ODS-2 $(5 \mu \mathrm{m}, 4.6 \mathrm{~mm} \times 250 \mathrm{~mm})$ was also used for separation. However, the time was longer, and the peak shapes are a bit broad. Thus, the ODS-3 $(4 \mu \mathrm{m}, 4.0 \mathrm{~mm} \times 150 \mathrm{~mm})$ was better to separate the compounds in excellent resolution.

The stock solution containing 27 standards was diluted to nine different concentrations. All diluted concentrations were injected to HPLC in triplicate. The concentration versus peak area for each 
standard in the mixture was plotted to obtain the calibration curve. Except for some of the compounds, the square of the correlation coefficient at $254 \mathrm{~nm}$ was more than $\mathrm{R}^{2}>0.99$, which indicates the linearity. At both 280 and $330 \mathrm{~nm}$, however, the square of correlation coefficients was more than $\mathrm{R}^{2}>0.999$.

$$
\text { Recovery } \%=\frac{\text { Measured conncentration-endegeneus concentration }}{\text { spiked concentration }} \times 100
$$

The recovery rates were in the range of $97-104 \%$ for each standard at $254 \mathrm{~nm}$ while $96-102 \%$ for each standard at 280 and $330 \mathrm{~nm}$ wavelengths. The recovery values indicate the efficacy and consistency of the method. The detection and quantification limits were calculated according to the general formula:

$$
\begin{aligned}
& \text { LOD : } 3.3(\sigma \sigma) / S S \\
& \text { LOQ : } 10(\sigma \sigma) / S S
\end{aligned}
$$

Where $(\sigma \sigma)$ is standard deviation of peak area, while $S S$ is the slope of the calibration curve [15].

The percentage relative standard deviation (RSD\%) indicating the degree of proximity of precision of the retention time, and the peak area was calculated. A known concentration $(20 \mu \mathrm{g} / \mathrm{mL})$ of the solution of mixed standards was injected into the HPLC system to check the repeatability of the retention time and peak areas $(\mathrm{Pa})$. Then RSD of peak areas of each retention time were calculated using seven replicate determinations.

\subsection{Calibration curves}

Standards compounds were dissolved in methanol to prepare stock solutions. Then retention times were detected by injecting each standard item individually. The mixture of 27 standard phenolic compounds was prepared in methanol and then injected into the HPLC-DAD. For quantitative analysis, six different concentrations of the twenty-seven phenolic compounds mixtures were injected triplicate. Then the calibration curves were obtained by plotting the concentration versus the peak areas of every compound. $R^{2}$ values were greater than 0.992 except $p$-benzoquinone, $R^{2}=0.983$; caffeic acid, $R^{2}=0.988$; ellagic acid $R^{2}=0.988$ (Table 2).

\section{Results and Discussion}

\subsection{Optimization of Chromatographic Condition}

In order to determine optimum chromatographic conditions, experiments were carried out under different mobile phase conditions. Acetonitrile, methanol, acetonitrile-acetic acid, and methanol aceticacid mobile phases by changing the acetic acid ratio were used to find the best resolution. Finally, the best mobile phase for separation was under the conditions of acetic-acid $0.1 \%$ in water as a solvent $\mathrm{A}$ and methanol as a solvent B. In many studies, acetonitrile use was suggested as one of the mobile phases [5,7,20-23]. Acetonitrile was also used for separation in this study. However, only 14 compounds, namely, gallic acid, protocatechuic acid, pyrocatechol, 2,4-dihydroxy phenyl ethanol, 4-hydroxy benzoic acid, vanillic acid, vanillin, $p$-coumaric acid, ferulic acid, trans-2-hydroxycinnamic acid, coumarin, cinnamic acid, naringenin, and chrysin could be separated. Therefore, it was aimed to change the solvent system to analyze more phenolic components at the same time. For this purpose, Inertsil ODS-3 $(4 \mu \mathrm{m}, 4.0 \mathrm{~mm} x$ $150 \mathrm{~mm}$ ) analytical column was used to see sharp peaks and to reduce the elution time. The column temperature was kept at $35^{\circ} \mathrm{C}$. With this optimized method, 27 phenolic components were analyzed with high accuracy and absolutely within 61 minutes.

\subsection{Method Validation}

In order to obtain separated peaks and optimum chromatograms with peak intensities close to each other, $500 \mu \mathrm{g} / \mathrm{mL}$ solutions were prepared from each standard and injected into the HPLC-DAD system. The location and peak intensities of each phenolic compound from the symmetrical and sharp peaks were determined. Then, concentrations of the compounds to be included in the mixture were 
determined (Figure 1). In order to completely separate the peaks, a gradient program was done. The linear gradient elution was provided by increasing the methanol ratio from $2 \%$ to $100 \%$. The phenolic mixture was prepared in methanol with amounts of standard compounds to test the linearity of the method.

The prepared mixture was diluted as a stock solution. From the stock solution, six standard dilutions were prepared, and each standard dilution was injected three times into HPLC-DAD under the same conditions (Figure 2). Calibration curves were created by determining the mean peak areas against the concentration of each dilution concentrations obtained from the chromatograms. Each standard was analyzed three times to increase the accuracy of the method, and the average values were calculated. Equations and $R^{2}$ values obtained for linear calibration curves are given in Table 2a, 2b, 2c. Table 2a contains the validation parameters of 27 components at $254 \mathrm{~nm}$ wavelength. As seen Table $2 \mathrm{a}, R^{2}$ values are above 0.992 except $p$-benzoquinone, $\left(R^{2}=0.983\right)$, caffeic acid $\left(R^{2}=0.988\right)$, and ellagic acid $\left(R^{2}=\right.$ 0.988). Table $2 \mathrm{~b}$ shows the calculated validation parameters of 19 phenolic compounds at $280 \mathrm{~nm}$ wavelength. All $R^{2}$ values are above 0.999 . In Table $2 \mathrm{c}$, the validation parameters of 11 compounds were calculated at $330 \mathrm{~nm}$ wavelength $\left(R^{2}>0.999\right)$.

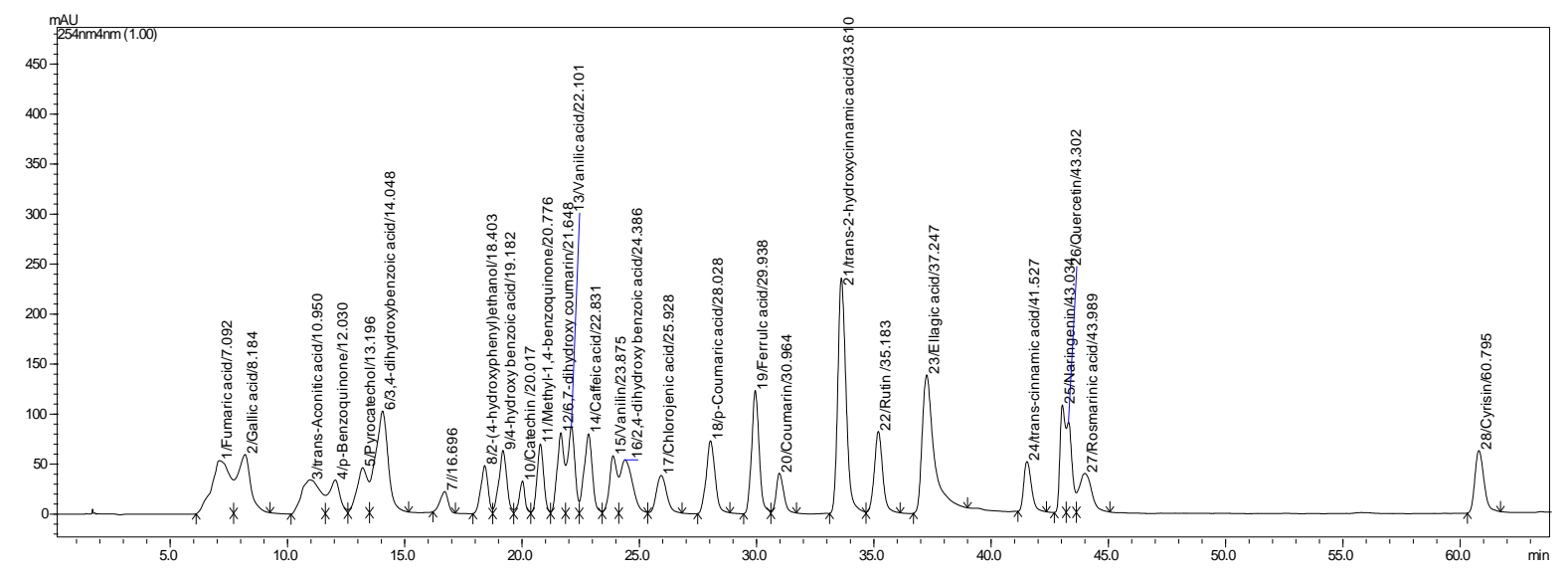

Figure 1. HPLC-DAD chromatograms of 27 phenolic standards at $254 \mathrm{~nm}$ : (1) fumaric acid, (2) gallic acid, (3) trans-aconitic acid, (4) $p$-benzoquinone, (5) pyrocatechol, (6), protocatechuic acid, (7) unknown, (8) 2-(4-hydroxyphenyl) ethanol, (9) 4-hydroxybenzoic acid, (10) (+)-catechin, (11) methyl-pbenzoquinone, (12) 6,7-dihydroxy coumarin, (13) vanillic acid, (14) caffeic acid, (15) vanillin, (16) 2,4dihydroxybenzoic acid, (17) chlorogenic acid, (18) $p$-coumaric acid, (19) ferulic acid, (20) coumarin, (21) trans-2-hydroxycinnamic acid, (22) rutin, (23) ellagic acid, (24) trans-cinnamic acid, (25) naringenin, (26) quercetin, (27) rosmarinic acid, (28) chrysin. Inertsil ODS-3 column (4 $\mu \mathrm{m}, 4 \mathrm{~mm}$ x $150 \mathrm{~mm})$. Mobile phase $0.1 \%$ acetic acid-methanol (gradient elution). Flow rate $1 \mathrm{~mL} / \mathrm{min}$. Diode array detection $254 \mathrm{~nm}$.

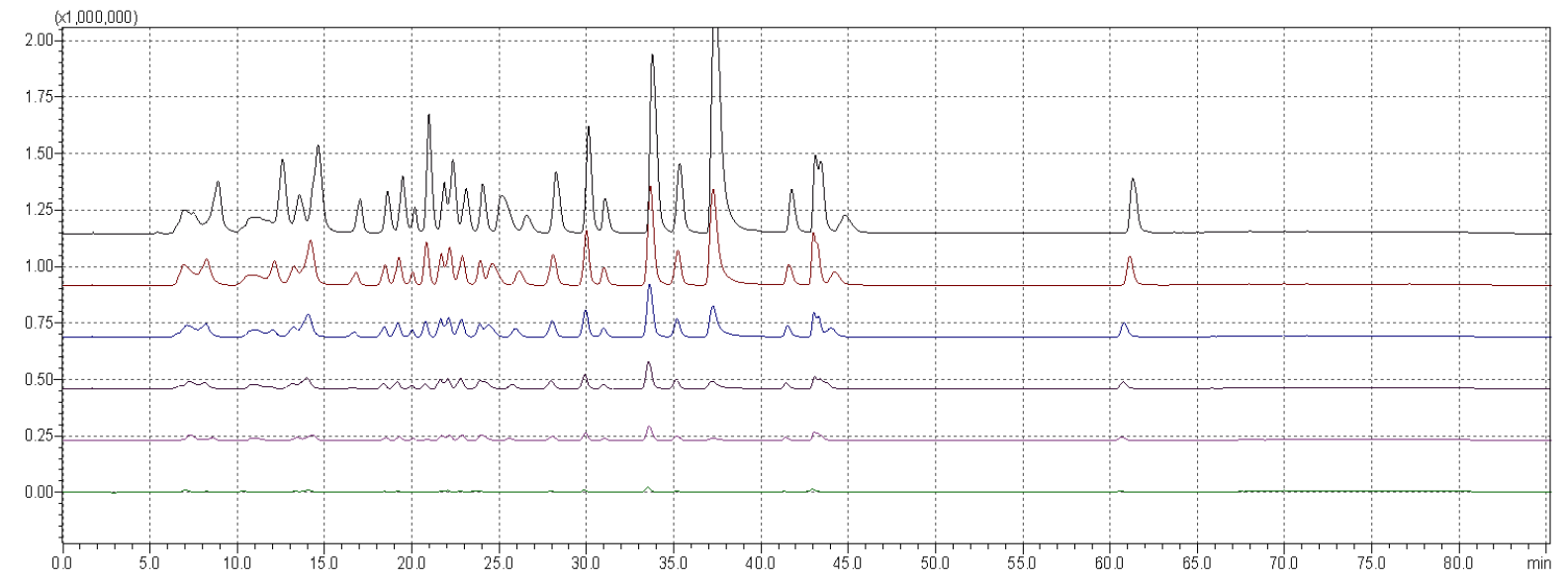

Figure 2. HPLC-DAD chromatograms of calibration standards 
Table 2a. Retention time, calibration curves, regression coefficient $\left(\mathrm{R}^{2}\right)$, linearity ranges, LODs and recoveries of phenolic standards at $254 \mathrm{~nm}$.

\begin{tabular}{|c|c|c|c|c|c|c|c|c|c|c|c|}
\hline No & Compounds & $\begin{array}{l}\mathbf{R T}^{\mathbf{a}} \\
(\mathbf{m i n})\end{array}$ & $\begin{array}{c}\text { Calibration } \\
\text { equation }\end{array}$ & $R^{2 \mathrm{~b}}$ & $\begin{array}{c}\text { Linear } \\
\text { range } \\
(\mu \mathrm{g} / \mathrm{mL})\end{array}$ & $\begin{array}{c}\lambda \max , \\
\mathbf{n m}\end{array}$ & $\begin{array}{c}\text { LOD }^{c} \\
(\mu \mathrm{g} / \mathrm{mL})\end{array}$ & $\begin{array}{c}\mathrm{LOQ}^{\mathrm{c}} \\
(\mu \mathrm{g} / \mathrm{mL})\end{array}$ & $\begin{array}{c}\text { Recovery } \\
(\%)\end{array}$ & $\begin{array}{c}\text { RSD }^{d} \\
\text { within day } \\
(n=7)\end{array}$ & $\begin{array}{c}\text { RSD } \\
\text { between } \\
\text { days }(n=7)\end{array}$ \\
\hline 1 & Fumaric acid & 6.81 & $y=11584 x-45691$ & 0.9970 & $7.5-125$ & 254 & 12.91 & 39.13 & $102.45 \pm 6.96$ & 4.96 & 2.20 \\
\hline 2 & Gallic acid & 8.39 & $\mathrm{y}=45439 x-137402$ & 0.9950 & $6.25-200$ & 254 & 2.23 & 6.77 & $102.46 \pm 4.46$ & 3.50 & 1.87 \\
\hline 3 & trans-Aconitic acid & 10.95 & $y=8314.5 x+57114$ & 0.9920 & $25.0-400$ & 254 & 6.12 & 18.54 & $101.16 \pm 8.11$ & 4.50 & 2.69 \\
\hline 4 & $p$-Benzoquinone & 12.62 & $y=39428 x-759865$ & 0.9970 & $50.0-200$ & 254 & 28.81 & 87.29 & $100.96 \pm 2.97$ & 4.88 & 2.17 \\
\hline 5 & Pyrocatechol & 13.35 & $y=5269.6 x+40422$ & 0.9950 & $31.3-500$ & 254 & 4.78 & 14.48 & $103.07 \pm 5.46$ & 5.44 & 1.40 \\
\hline 6 & Protocatechuic acid & 14.10 & $y=76181 x-88801$ & 0.9995 & $3.13-100$ & 254 & 3.42 & 10.35 & $102.35 \pm 4.21$ & 3.19 & 1.22 \\
\hline 7 & 2-(4-Hydroxyphenyl)-ethanol & 18.40 & $y=2907.6 x-7629.4$ & 0.9995 & $21.3-1366$ & 254 & 2.77 & 8.39 & $102.32 \pm 3.57$ & 3.39 & 1.27 \\
\hline 8 & 4-Hydroxybenzoic acid & 19.50 & $y=111102 x+21691$ & 0.9993 & $1.56-50.0$ & 254 & 1.58 & 4.79 & $100.82 \pm 3.89$ & 4.00 & 2.41 \\
\hline 9 & $(+)$-Catechin & 20.00 & $y=3865.1 x+32660$ & 0.9980 & $15.6-500$ & 254 & 3.29 & 9.96 & $102.11 \pm 4.08$ & 3.78 & 2.87 \\
\hline 10 & Methyl-p-benzoquinone & 20.83 & $y=81195 x-420112$ & 0.9830 & $3.13-50.0$ & 254 & 5.37 & 16.27 & $103.03 \pm 4.23$ & 4.86 & 5.29 \\
\hline 11 & 6,7-Dihydroxycoumarin & 21.99 & $y=34377 x-32740$ & 0.9940 & $5.00-50$ & 254 & 3.98 & 12.07 & $104.11 \pm 5.06$ & 4.94 & 3.72 \\
\hline 12 & Vanillic acid & 22.37 & $y=74653 x-9634.1$ & 0.9998 & $1.56-100$ & 254 & 1.56 & 4.68 & $103.58 \pm 4.43$ & 5.06 & 3.88 \\
\hline 13 & Caffeic acid & 22.94 & $y=67972 x-32965$ & 0.9880 & $3.00-30.0$ & 254 & 4.54 & 13.75 & $102.67 \pm 4.92$ & 4.01 & 5.87 \\
\hline 14 & Vanillin & 24.02 & $y=45495 x+313074$ & 0.9920 & $3.13-100$ & 254 & 1.21 & 3.67 & $100.59 \pm 4.84$ & 1.76 & 3.76 \\
\hline 15 & 2,4-Dihydroxybenzoic acid & 24.78 & $y=70870 x+258749$ & 0.9920 & $1.56-100$ & 254 & 4.17 & 12.63 & $100.35 \pm 4.75$ & 5.10 & 5.81 \\
\hline 16 & Chlorogenic acid & 26.13 & $y=39264 x+66133$ & 0.9920 & $6.25-50.0$ & 254 & 6.25 & 18.75 & $101.23 \pm 4.98$ & 5.20 & 3.53 \\
\hline 17 & $p$-Coumaric acid & 28.43 & $y=18300 x+6153.3$ & 0.9998 & $6.25-400$ & 254 & 5.46 & 16.56 & $101.60 \pm 2.36$ & 3.14 & 0.44 \\
\hline 18 & Ferulic acid & 29.93 & $y=35737 x+12977$ & 0.9999 & $2.34-300$ & 254 & 3.96 & 11.99 & $100.99 \pm 3.54$ & 3.20 & 0.51 \\
\hline 19 & Coumarin & 31.10 & $y=36021 x-23215$ & 0.9999 & $3.13-100$ & 254 & 2.21 & 6.69 & $101.74 \pm 4.83$ & 3.59 & 1.08 \\
\hline 20 & $\begin{array}{l}\text { trans-2-Hydroxycinnamic } \\
\text { acid }\end{array}$ & 33.65 & $y=53843 x+124308$ & 0.9996 & $3.13-400$ & 254 & 3.09 & 9.27 & $99.75 \pm 3.75$ & 2.85 & 0.75 \\
\hline 21 & Rutin & 35.02 & $y=40347 x-30437$ & 0.9999 & $3.13-200$ & 254 & 2.49 & 7.56 & $101.99 \pm 3.45$ & 3.01 & 2.53 \\
\hline 22 & Ellagic acid & 37.61 & $y=165729 x-3000000$ & 0.9880 & $12.5-100$ & 254 & 2.68 & 8.13 & $100.70 \pm 5.43$ & 7.34 & 6.56 \\
\hline 23 & trans-Cinnamic acid & 41.54 & $\mathrm{y}=87505+4540.2$ & 0.9999 & $1.25-50.0$ & 254 & 0.58 & 1.74 & $100.85 \pm 1.58$ & 5.78 & 5.66 \\
\hline 24 & Naringenin & 43.07 & $y=9895.8 x+159212$ & 0.9950 & $4.84-620$ & 254 & 2.75 & 8.34 & $100.00 \pm 4.91$ & 2.88 & 2.39 \\
\hline 25 & Quercetin & 43.49 & $y=68024 x+7902.3$ & 0.9999 & $1.56-100$ & 254 & 1.01 & 3.06 & $98.57 \pm 3.84$ & 1.87 & 5.04 \\
\hline 26 & Rosmarinic acid & 44.20 & $y=20734 x+853142$ & 0.9950 & $30.0-120$ & 254 & 6.38 & 19.33 & $97.92 \pm 4.36$ & 6.12 & 4.97 \\
\hline 27 & Chrysin & 60.45 & $y=66794 x-17157$ & 0.9999 & $3.13-100$ & 254 & 1.96 & 5.93 & $101.35 \pm 3.17$ & 3.64 & 1.11 \\
\hline
\end{tabular}

${ }^{\mathrm{a}} \mathrm{RT}$ : Retention time of the compound in minutes, ${ }^{\mathrm{b}} R^{2}$ : linearity of the calibration graph, ${ }^{\mathrm{c}}$ LOD: Limit of Detection in $\mu \mathrm{g} / \mathrm{m}$ Land LOQ: Limit of Quantification in $\mu \mathrm{g} / \mathrm{mL},{ }^{\mathrm{d}} \mathrm{RSD}$ : Percentage relative standard deviation 
Table 2b. Retention time, calibration curves, regression coefficient $\left(\mathrm{R}^{2}\right)$, linearity ranges, LODs and recoveries of phenolic standards at $280 \mathrm{~nm}$.

\begin{tabular}{|c|c|c|c|c|c|c|c|c|c|c|c|}
\hline No & Phenolic Compounds & $\begin{array}{l}\mathbf{R T}^{\mathrm{a}} \\
(\mathbf{m i n})\end{array}$ & Calibration equation & $R^{2 \mathrm{~b}}$ & $\begin{array}{c}\text { Linear } \\
\text { range } \\
(\mu \mathrm{g} / \mathrm{mL})\end{array}$ & $\begin{array}{c}\lambda_{\max }, \\
\mathbf{n m}\end{array}$ & $\begin{array}{c}\text { LOD }^{c} \\
(\mu \mathrm{g} / \mathrm{mL})\end{array}$ & $\begin{array}{c}\text { LOQ }^{c} \\
(\mu \mathrm{g} / \mathrm{mL})\end{array}$ & $\begin{array}{c}\text { Recovery } \\
(\%)\end{array}$ & $\begin{array}{c}\text { RSD }^{d} \\
\text { within } \\
\text { days } \\
(n=7)\end{array}$ & $\begin{array}{c}\text { RSD } \\
\text { between } \\
\text { days }(n=7)\end{array}$ \\
\hline 1 & Pyrocatechol & 13.35 & $y=20632 x+53090$ & 0.9999 & $7.8-250$ & 280 & 4.72 & 14.32 & $100.35 \pm 2.07$ & 3.51 & 3.20 \\
\hline 2 & Protocatechuic acid & 14.10 & $y=33573 x+21157$ & 0.9995 & $6.25-200$ & 280 & 6.25 & 18.75 & $100.12 \pm 3.05$ & 2.41 & 6.10 \\
\hline 3 & 2-(4-Hydroxyphenyl) ethanol & 18.40 & $y=11284 x+95007$ & 0.9999 & $10.6-341$ & 280 & 5.60 & 16.2 & $98.99 \pm 2.25$ & 2.03 & 4.15 \\
\hline 4 & 4-hydroxybenzoic acid & 19.50 & $y=30844 x+18883$ & 0.9998 & $1.25-50$ & 280 & 1.33 & 4.03 & $99.07 \pm 3.97$ & 4.43 & 4.38 \\
\hline 5 & $(+)$-Catechin & 20.00 & $y=14456 x+37858$ & 0.9999 & $3.9-500$ & 280 & 0.95 & 2.86 & $101.25 \pm 2.16$ & 4.00 & 3.82 \\
\hline 6 & Methyl- $p$-benzoquinone & 20.83 & $y=2566.8 x+14266$ & 0.9998 & $3.13-50$ & 280 & 1.45 & 4.40 & $100.11 \pm 1.42$ & 25.8 & 4.90 \\
\hline 7 & 6,7-Dihydroxycoumarin & 21.99 & $y=21346 x+25710$ & 0.9992 & $1.56-200$ & 280 & 0.92 & 2.76 & $104.06 \pm 4.08$ & 7.40 & 6.11 \\
\hline 8 & Vanillic acid & 22.37 & $y=36710 x+2454.3$ & 0.9990 & $0.78-100$ & 280 & 0.54 & 1.62 & $102.13 \pm 4.64$ & 3.59 & 4.55 \\
\hline 9 & Caffeic acid & 22.94 & $y=46945 x+12850$ & 0.9995 & $0.94-120$ & 280 & 0.23 & 0.69 & $101.36 \pm 5.19$ & 2.80 & 4.83 \\
\hline 10 & Vanillin & 24.02 & $y=215103 x+89945$ & 0.9997 & $0.78-25$ & 280 & 0.75 & 2.28 & $101.80 \pm 3.81$ & 6.82 & 7.84 \\
\hline 11 & $p$-Coumaric acid & 28.43 & $y=96659 x+175014$ & 0.9999 & $3.13-400$ & 280 & 3.08 & 9.24 & $98.48 \pm 7.84$ & 1.59 & 3.19 \\
\hline 12 & Ferulic acid & 29.93 & $y=63874 x-3785.8$ & 0.9997 & $2.34-300$ & 280 & 1.62 & 4.92 & $102.29 \pm 4.96$ & 0.88 & 3.65 \\
\hline 13 & Coumarin & 31.10 & $y=45026 x+56387$ & 0.9995 & $0.78-25$ & 280 & 0.93 & 2.82 & $99.25 \pm 5.13$ & 1.81 & 3.21 \\
\hline 14 & trans-2-Hydroxycinnamic acid & 33.65 & $y=123059 x+435116$ & 0.9996 & $6.25-200$ & 280 & 6.25 & 18.95 & $97.47 \pm 4.30$ & 1.62 & 3.00 \\
\hline 15 & Rutin & 35.02 & $y=15485 x-919.6$ & 0.9998 & $1.56-200$ & 280 & 4.00 & 12.00 & $101.28 \pm 3.35$ & 4.79 & 3.58 \\
\hline 16 & trans-Cinnamic acid & 41.54 & $y=174388 x+79400$ & 0.9997 & $1.25-50$ & 280 & 1.43 & 4.35 & $97.62 \pm 4.49$ & 4.12 & 3.27 \\
\hline 17 & Naringenin & 43.07 & $y=72633 x+837946$ & 0.9996 & $4.84-155$ & 280 & 5.01 & 15.03 & $100.58 \pm 4.12$ & 1.87 & 4.25 \\
\hline 18 & Quercetin & 43.49 & $y=420803 x+735567$ & 0.9998 & $3.13-100$ & 280 & 2.83 & 8.58 & $101.90 \pm 3.92$ & 2.60 & 6.28 \\
\hline 19 & Chrysin & 60.45 & $y=63792 x+41075$ & 0.9999 & $1.56-100$ & 280 & 1.51 & 4.59 & $98.61 \pm 4.93$ & 2.47 & 3.00 \\
\hline
\end{tabular}

${ }^{\mathrm{a}} \mathrm{RT}$ : Retention time of the compound in minutes

${ }^{\mathrm{b}} R^{2}$ : linearity of the calibration graph

${ }^{c}$ LOD: Limit of Detection in $\mu \mathrm{g} / \mathrm{m}$ Land LOQ: Limit of Quantification in $\mu \mathrm{g} / \mathrm{mL}$

${ }^{\mathrm{d}} \mathrm{RSD}$ : Percentage relative standard deviation 
Table 2c. Retention time, calibration curves, regression coefficient $\left(\mathrm{R}^{2}\right)$, linearity ranges, LODs and recoveries of phenolic standards at $330 \mathrm{~nm}$.

\begin{tabular}{|c|c|c|c|c|c|c|c|c|c|c|c|}
\hline No & Phenolic Compounds & $\begin{array}{l}\mathrm{RT}^{\mathrm{a}} \\
(\mathrm{min})\end{array}$ & Calibration equation & $R^{2 \mathrm{~b}}$ & $\begin{array}{c}\text { Linear } \\
\text { range } \\
(\mu \mathrm{g} / \mathrm{mL})\end{array}$ & $\begin{array}{c}\lambda_{\max }, \\
\mathbf{n m}\end{array}$ & $\begin{array}{c}\text { LOD }^{c} \\
(\mu \mathrm{g} / \mathrm{mL})\end{array}$ & $\begin{array}{c}\mathrm{LOQ}^{\mathrm{c}} \\
(\mu \mathrm{g} / \mathrm{mL})\end{array}$ & $\begin{array}{c}\text { Recovery } \\
(\%)\end{array}$ & $\begin{array}{c}\text { RSD }^{d} \\
\text { within } \\
\text { days }(n= \\
7)\end{array}$ & $\begin{array}{c}\text { RSD } \\
\text { between } \\
\text { days } \\
(\mathbf{n}=7) \\
\end{array}$ \\
\hline 1 & 6,7-Dihydroxycoumarin & 21.99 & $y=54384 x-77273$ & 0.9998 & $1.56-200$ & 330 & 3.89 & 11.81 & $102.77 \pm 4.70$ & 2.90 & 4.56 \\
\hline 2 & Caffeic acid & 22.94 & $y=75745 x-36431$ & 0.9993 & $0.94-60$ & 330 & 2.76 & 8.36 & $101.66 \pm 5.78$ & 5.53 & 3.36 \\
\hline 3 & Vanillin & 24.02 & $y=74432 x+43718$ & 0.9998 & $1.56-100$ & 330 & 2.06 & 6.02 & $99.41 \pm 3.67$ & 29.89 & 4.31 \\
\hline 4 & $p$-Coumaric acid & 28.43 & $y=286585 x+162918$ & 0.9998 & $1.56-100$ & 330 & 2.14 & 6.47 & $96.90 \pm 10.27$ & 1.53 & 3.52 \\
\hline 5 & Ferulic acid & 29.93 & $y=104472 x+105130$ & 0.9999 & $4.70-300$ & 330 & 3.99 & 12.11 & $99.79 \pm 4.51$ & 1.06 & 3.77 \\
\hline 6 & Coumarin & 31.10 & $y=29083 x+1992.1$ & 0.9999 & $1.56-100$ & 330 & 1.44 & 4.36 & $99.64 \pm 5.76$ & 2.19 & 3.20 \\
\hline 7 & trans-2-Hydroxycinnamic acid & 33.65 & $y=67576 x+162187$ & 1.000 & $3.13-400$ & 330 & 2.99 & 9.06 & $99.42 \pm 1.73$ & 1.58 & 3.08 \\
\hline 8 & Rutin & 35.02 & $y=25414 x+35748$ & 0.9997 & $1.56-200$ & 330 & 5.76 & 17.44 & $97.82 \pm 4.53$ & 2.16 & 4.52 \\
\hline 9 & Ellagic acid & 37.61 & $y=18964 x-109643$ & 0.9998 & $6.25-200$ & 330 & 5.60 & 16.97 & $101.27 \pm 4.60$ & 6.05 & 8.43 \\
\hline 10 & trans-Cinnamic acid & 41.54 & $y=129885 x+78457$ & 1.0000 & $1.56-100$ & 330 & 0.98 & 2.97 & $99.93 \pm 4.08$ & 4.24 & 5.65 \\
\hline 11 & Chrysin & 60.45 & $y=42937 x+1034.7$ & 0.9998 & $1.56-100$ & 330 & 1.60 & 4.80 & $100.39 \pm 4.27$ & 2.75 & 3.11 \\
\hline
\end{tabular}

${ }^{a}$ RT: Retention time of the compound in minutes

${ }^{\mathrm{b}} R^{2}$ : linearity of the calibration graph

${ }^{c}$ LOD: Limit of Detection in $\mu \mathrm{g} / \mathrm{m}$ Land LOQ: Limit of Quantification in $\mu \mathrm{g} / \mathrm{mL}$

${ }^{\mathrm{d}}$ RSD: Percentage relative standard deviation 
Repeatability of the method throughout a day and between days of a standard solution mixture consist of fumaric acid $(125 \mu \mathrm{g} / \mathrm{mL})$, gallic acid $(25 \mu \mathrm{g} / \mathrm{mL})$, trans-aconitic acid $(100 \mu \mathrm{g} / \mathrm{mL}), p$ benzoquinone $(25 \mu \mathrm{g} / \mathrm{mL})$, pyrocatechol $(125 \mu \mathrm{g} / \mathrm{mL})$, protocatechuic acid $(25 \mu \mathrm{g} / \mathrm{mL}, 2-(4-$ hydroxyphenyl) ethanol $(170 \mu \mathrm{g} / \mathrm{mL})$, 4-hydroxybenzoic acid $(6.25 \mu \mathrm{g} / \mathrm{mL}),(+)$-catechin $(62.5 \mu \mathrm{g} / \mathrm{mL})$, methyl-p-benzoquinone (12.5 $\mu \mathrm{g} / \mathrm{mL}), 6,7$-dihydroxy coumarin $(25 \mu \mathrm{g} / \mathrm{mL})$, vanillic acid $(12.5 \mu \mathrm{g} / \mathrm{mL})$, caffeic acid $(15.0 \mu \mathrm{g} / \mathrm{mL})$, vanillin $(12.5 \mu \mathrm{g} / \mathrm{mL}), 2,4$-dihydroxybenzoic acid $(12.5 \mu \mathrm{g} / \mathrm{mL})$, chlorogenic $\operatorname{acid}(12.5 \mu \mathrm{g} / \mathrm{mL}), p$-coumaric acid $(50 \mu \mathrm{g} / \mathrm{mL})$, ferulic acid $(37.5 \mu \mathrm{g} / \mathrm{mL})$, coumarin $(12.5 \mu \mathrm{g} / \mathrm{mL})$, trans2-hydroxycinnamic acid $(50 \mu \mathrm{g} / \mathrm{mL})$, rutin $(25 \mu \mathrm{g} / \mathrm{mL})$, ellagic acid $(25 \mu \mathrm{g} / \mathrm{mL})$, trans-cinnamic acid $(6.25 \mu \mathrm{g} / \mathrm{mL})$, naringenin $(77.5 \mu \mathrm{g} / \mathrm{mL})$, quercetin $(12.5 \mu \mathrm{g} / \mathrm{mL})$, rosmarinic acid $(15 \mu \mathrm{g} / \mathrm{mL})$, chrysin $(12.5 \mu \mathrm{g} / \mathrm{mL}$, verified by injecting into HPLC-DAD seven times. Relative standard deviation values were calculated from the data obtained by seven times repeating during the day and between days. These were used to determine the repeatability. As seen in Table 2a, the repeatability of the method is 1.76-7.34 RSD during the day and 0.44-6.56 RSD between the days. However, except for ellagic acid and rosmarinic acid during the day, the repeatability of other compounds is below $6 \%$. Repeatability is higher for compounds rather than ellagic acid between days. RSD was between 0.88-4.79\% (excluding methyl-p-benzoquinone, vanillin, and 6,7-dihydroxy coumarin), as given in Table 2b and RSD 1.06-5.53 (excluding ellagic acid, vanillin) as given in Table $2 \mathrm{c}$. The RSD values were coherent with those of studies in the literature [9$10]$.

The precision of the method was also calculated using the equations obtained from the calibration curve as a result of six repeated analyses. The above mentioned standard mixture of compounds was injected six times. Then the calibration curve and the concentrations of the compounds were calculated using the peak areas obtained from the chromatograms. The obtained concentration values were compared with actual values to determine the recovery and, therefore, the precision of the method. At $254 \mathrm{~nm}$, the recovery of the method is in the range of $97.92 \pm 4.36-103.07 \pm 5.46$ as given in Table $2 \mathrm{a}$, while at 280 $\mathrm{nm} 97.47 \pm 4.30-104.06 \pm 4.08$ in Table $2 \mathrm{~b}$. At $330 \mathrm{~nm}$, however, it is $96.90 \pm 10.27-102.77 \pm 4.70$, as given in Table 2c. Therefore, it is possible to say that the accuracy of the optimized method is quite high. Besides, LOD values of the method were determined and given in Tables $2 \mathrm{a}, 2 \mathrm{~b}$, and $2 \mathrm{c}$. The obtained values provide accuracy and low detection of the compounds using HPLC in a cheaper and faster way.

\section{Conclusions}

A practical, fast rapid, and accurate RP-HPLC-DAD method was developed to analyze twenty-seven natural compounds simultaneously in one injection. The total analyzing time was 61 minutes. Developed method validation was performed using linearity, relative error, reproducibility, LOD, and LOQ values. The method exhibited excellent linearity, good precision, and recovery. Various wavelengths, such as $220 \mathrm{~nm}, 280 \mathrm{~nm}$, and $330 \mathrm{~nm}$, were used to detect the compounds for high accuracy. Since the synthetic drugs have many side effects, the researchers start to investigate the natural products to find effective drugs. Some of the natural products studied are the potential drugs since possessing adequate in vitro and in vivo activities. However, it is tough to be approved of any effective natural product as a drug. Therefore, entrepreneurs or companies get permission to be able to sell these products as dietary supplements. If the productions of food supplements are produced by the following their production procedure, there is no problem. However, some of the food traders and malicious people who want to make more money can adulterate food supplements, then which will be dangerous for human health. Therefore, commercial food supplements are necessary to be analyzed for their ingredients. The developed method can be used for any natural product analyses and any commercial food supplement related to twenty-seven compounds. 


\section{Acknowledgments}

The paper has been granted by the Muğla Sitk1 Koçman University Research Projects Coordination Office through Project Grant Number: (15/154) and Project Grand Number: (17/216). One of us (C.Ç.) thanks the Higher Education Commission of Turkey (YOK) for the YOK 100/2000 thematic scholarship.

\section{Supporting Information}

Supporting information accompanies this paper on http://www.acgpubs.org/journal/journal-ofchemical-metrology

\section{ORCID}

Özge Tokul-Ölmez: 0000-0002-2093-4949

Bihter Şahin: 0000-0001-8657-052X

Cansel Çakır: 0000-0002-6175-9008

Mehmet Öztürk: 0000-0001-8932-4535

\section{References}

[1] M. Fotovvat, T. Radjabian and A. Saboora (2019). HPLC fingerprint of important phenolic compounds in some Salvia L. species from Iran, Rec. Nat. Prod. 13(1), 37-49.

[2] H. Han, H. Yılmaz and İ. Gülçin (2018). Antioxidant activity of flaxseed (Linum usitatissimum L.) shell and analysis of its polyphenol contents by LC-MS/MS, Rec. Nat. Prod. 12(4), 397-402.

[3] T. Sabudak, M. Ozturk and E. Alpay (2017). New bioflavonoids from Solanum nigrum L. by anticholinesterase and anti-tyrosinase activities-guided fractionation, Rec. Nat. Prod. 11(2), 130-140.

[4] L. Yang, Z. Wang and L. Xu (2006). Simultaneous determination of phenols (bibenzyl, phenanthrene, and fluorenone) in Dendrobium species by high-performance liquid chromatography with diode array detection, J. Chromatogr. A 1104, 230-237.

[5] M. G. Carvalho, C. F. S Aragão, F. N. Raffin and T. F. A. de L. Moura (2017). Development and validation of a simultaneous RP-HPLC-UV/DAD method for determination of polyphenols in gels containing $S$. terebinthifolius Raddi (Anacardiaceae), Pharmacogn. Mag. 13(50), 309-315.

[6] C. A. Ballus, A. D. Meinhart, R. E. Bruns and H. T.Godoy (2011). Use of multivariate statistical techniques to optimize the simultaneous separation of 13 phenolic compounds from extra-virgin olive oil by capillary electrophoresis, Talanta 83, 1181-1187.

[7] M. L. dL. Francisco, A.V.A. Resurreccion and A.V.A. Resurreccion(2009). Development of a reversedphase high-performance liquid chromatography (RP-HPLC) procedure for the simultaneous determination of phenolic compounds in peanut skin extracts, Food Chem. 117(2), 356-363.

[8] L. M. Crawford, D. M. Holstege and S. C. Wang (2018). High-throughput extraction method for phenolic compounds in olive fruit (Olea europaea), J. Food Compos. Anal. 66, 136-144.

[9] M. M. P. Natividade, L. C. Corrêa, S. V. C. de Souza, G. E. Pereir and L.C. de Oliveira Lima (2013). Simultaneous analysis of 25 phenolic compounds in grape juice for HPLC: Method validation and characterization of São Francisco Valley samples, Microchem. J. 110, 665-674.

[10] C. V. da S. Padilha, G. A. Miskinis, M. E. A. O. de Souza, G. E. Pereira, D. de Oliveira, M. T. BordignonLuiz and M. dos S. Lima (2017). Rapid determination of flavonoids and phenolic acids in grape juices and wines by RP-HPLC/DAD: Method validation and characterization of commercial products of the new Brazilian varieties of grape, Food Chem. 228, 106-115.

[11] I. Tomaz and L. Maslov (2016). Simultaneous determination of phenolic compounds in different matrices using phenyl-hexyl stationary phase, Food Anal. Method. 9,401-410.

[12] R. Aliyazicioglu, S. Demir, M. Badem, S.O. Sener, N. Korkmaz, E.A. Demir, U. Ozgen, S.A. Karaoglu, and Y. Aliyazicioglu (2017). Antioxidant, antigenotoxic, antimicrobial activities and phytochemical analysis of dianthus carmelitarum, Rec. Nat. Prod. 11(3), 270-284.

[13] I. Lazarova, G. Zengin, A. Aktumsek, R. Gevrenova, R. Ceylan and S. Uysal (2014). HPLC-DAD analysis of phenolic compounds and antioxidant properties of Asphodeline lutea roots from Bulgaria and Turkey, Ind. Crops Prod. 61, 438-441. 
[14] Y. Cai, Q. Luo, M. Sun and H. Corke (2004). Antioxidant activity and phenolic compounds of 112 traditional Chinese medicinal plants associated with anticancer, Life Sci. 74, 2157-2184.

[15] T. Seal (2016). HPLC determination of phenolic acids, flavonoids and ascorbic acid in four different solvent extracts of Zanthoxylum acanthopodium, a wild edible plant of Meghalaya State of India, Int. J. Pharm. Pharm. Sci. 8, 103-109.

[16] P. Costa, S. Gonçalves, P. Valentão, P. B. Andrade, N. Coelho and A. Romano (2012). Thymus lotocephalus wild plants and in vitro cultures produce different profiles of phenolic compounds with antioxidant activity, Food Chem. 135, 1253-1260.

[17] L. Peng, X. Song, X. Shi, J. Li and C. Ye (2008). An improved HPLC method for simultaneous determination of phenolic compounds, purine alkaloids and theanine in Camellia species, J. Food Comp. Anal. 21, 559-563.

[18] A. Č. Mišan, N. M. Mimica-Dukić, A. I. Mandić, M. B. Sakač, I. Lj. Milovanović and I. J. Sedej (2011). Development of a rapid resolution HPLC method for the separation and determination of 17 phenolic compounds in crude plant extracts, Cent. Eur. J. Chem. 9, 133-142.

[19] D. Kahoun, S. Rezková, K. Ves`krnová, J. Královsky`and M. Holčapek (2008). Determination of phenolic compounds and hydroxymethylfurfural in meads using high performance liquid chromatography with coulometric-array and UV detection, J. Chromatogr. A 1202, 19-33.

[20] A.H. Liu, L. Li, M.Xu, Y.H. Lin, H.Z. Guo and D.A. Guo (2006). Simultaneous quantification of six major phenolic acids in the roots of Salvia miltiorrhiza and four related traditional Chinese medicinal preparations by HPLC-DAD method, J. Pharm. Biomed. Anal. 41, 48-56.

[21] P. Mattila, J. Astola, and J. Kumpulainen (2000). Determination of flavonoids in plant material by HPLC with diode-array and electro-array detections, J. Agric. Food Chem. 48, 5834-5841.

[22] İ.E. Poyraz, G.A Çiftçi, and N. Öztürk (2017). Phenolic contents, in vitro antioxidant and cytotoxicity activities of Salvia aethiopis L. and S. ceratophylla L. (Lamiaceae), Rec. Nat. Prod. 11:4, 345-355.

[23] M.A. Rostagno, N. Manchón, M. D’Arrigo, E. Guillamón, A. Villares, A. García-Lafuente, A. Ramos and J.A. Martínez (2011). Fast and simultaneous determination of phenolic compounds and caffeine in teas, mate, instant coffee, soft drink and energetic drink by high-performance liquid chromatography using a fused-core column, Anal. Chim. Acta. 685, 204-211.

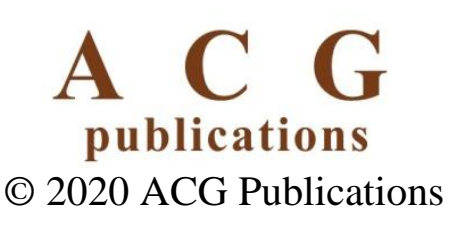

